\title{
ON DIFFERENTIABILITY OF MAPPINGS WITH FINITE DILATION
}

\author{
MaŁgorzata Turowska
}

\begin{abstract}
We study mappings $f:(a, b) \rightarrow Y$ with finite dilation having Lebesgue integrable majorant, where $Y$ is a real normed vector space. We construct Lipschitz mapping $f:(a, b) \rightarrow Y$, $\operatorname{dim} Y=\infty$, which is nowhere differentiable but its graph has everywhere trivial contingent. We show that if the contingent of the graph of a mapping with finite dilation is a nontrivial space, then $f$ is almost everywhere differentiable.
\end{abstract}

\section{Definitions and preliminaries}

Let $Y$ be a real normed space. Let $f:(a, b) \rightarrow Y$ be a mapping with finite dilation, i.e.,

$$
\forall x \in(a, b): \limsup _{h \rightarrow 0}\left\|\frac{f(x+h)-f(x)}{h}\right\|<\infty .
$$

If $\operatorname{dim} Y<\infty$, then it is well-known that $f$ is almost everywhere differentiable. In the case of infinite dimension of the space $Y$ this is no longer true. First, we will give auxiliary definitions and theorems required to prove the main theorem.

Definition 1.1 (9]). Let $\emptyset \neq M \subset Z$, where $Z$ is a normed space, and $z \in \operatorname{cl} M$. The set

$\left\{v \in Z: \exists\left(z_{n}\right)_{n \in \mathbb{N}}, z_{n} \in M, \lim _{n \rightarrow \infty} z_{n}=z, \exists\left(\lambda_{n}\right)_{n \in \mathbb{N}}, \lambda_{n}>0: \lim _{n \rightarrow \infty} \lambda_{n}\left(z_{n}-z\right)=v\right\}$

is called a tangent cone to $M$ at the point $z$ and is denoted $\operatorname{Tan}(M, z)$. Elements of $\operatorname{Tan}(M, z)$ are called vectors tangent to $M$ at $z$. The set $\operatorname{Tan}(M, z)$ is also called a contingent of $M$ at $z$ (for other definitions of $\operatorname{Tan}(M, z)$, see, e.g., 2, [4], [5], [8]).

(C) 2015 Mathematical Institute, Slovak Academy of Sciences. 2010 Mathematics Subject Classification: 26E20, 46 G05.

Keywords: contingent (tangent cone), differentiability, Pettis theorem, Bochner integrability. 
Let $X, Y$ be real normed spaces. $\operatorname{Gr}(\varphi)$ will denote the graph of a mapping $\varphi: E \rightarrow Y, E \subset X$, and $0_{Y}$ will stand for a zero vector of the space $Y$. We will need the following lemmas and definitions.

LEMmA $1.2([7)$. Let $X$ and $Y$ be real normed spaces and let $U \subset X$ be an open set. Let $F: U \rightarrow Y$ and $f: U \rightarrow Y$ be mappings continuous at a point $x_{0} \in U$. Assume that $F$ is differentiable at $x_{0}$ and $\operatorname{Tan}\left(\operatorname{Gr}(f),\left(x_{0}, f\left(x_{0}\right)\right)\right)$ is a linear subspace of $X \times Y$. Then, $\operatorname{Tan}\left(\operatorname{Gr}(F+f),\left(x_{0},(F+f)\left(x_{0}\right)\right)\right)$ is a linear subspace of $X \times Y$. Moreover,

$$
\begin{aligned}
\operatorname{Tan}\left(\operatorname{Gr}(F+f),\left(x_{0},(F+f)\left(x_{0}\right)\right)\right) & = \\
& \left\{\left(u, F^{\prime}\left(x_{0}\right) u+v\right):(u, v) \in \operatorname{Tan}\left(\operatorname{Gr}(f),\left(x_{0}, f\left(x_{0}\right)\right)\right)\right\} .
\end{aligned}
$$

Let $X \neq \emptyset, \Sigma$ be a $\sigma$-field of subsets of the space $X$ and let $\mu: \Sigma \rightarrow[0, \infty)$ be a measure. From now, let $Y$ be a Banach space and $X$ be a normed space with a finite measure $\mu$.

Definition $1.3([3])$. A function $\varphi: X \rightarrow Y$ is called a step function if there exist points $y_{1}, y_{2}, \ldots, y_{n}$ in $Y$ and measurable sets $E_{1}, E_{2}, \ldots, E_{n}$ such that

$$
\varphi=\sum_{i=1}^{n} y_{i} \chi_{E_{i}}, \quad \text { where } \quad \chi_{E_{i}}(x)= \begin{cases}1, & x \in E_{i}, \\ 0, & x \notin E_{i} .\end{cases}
$$

Definition 1.4 (3]). A function $\varphi: X \rightarrow Y$ is called $\mu$-measurable if there exists a sequence of step functions $\left(\varphi_{n}\right)_{n \in \mathbb{N}}$ such that $\lim _{n \rightarrow \infty}\left\|\varphi_{n}-\varphi\right\|=0$ $\mu$-almost everywhere $(\|\cdot\|$ is sup norm in the space of bounded functions).

Let $y^{*}: Y \rightarrow \mathbb{R}$ be a linear continuous functional on $Y$, i.e., $y^{*} \in Y^{*}$.

Definition 1.5 ([3]). A function $\varphi: X \rightarrow Y$ is called weakly $\mu$-measurable if $y^{*} \circ \varphi: X \rightarrow \mathbb{R}$ is $\mu$-measurable for each $y^{*} \in Y^{*}$.

Theorem 1.6 (6]). A necessary and sufficient condition that $\varphi: X \rightarrow Y$ is $\mu$-measurable is that $\varphi$ is weakly $\mu$-measurable and separably-valued (separably-valued means that the range $\varphi(X)$ is separable).

Definition 1.7 ([3]). Let $\varphi: X \rightarrow Y, \varphi=\sum_{i=1}^{n} y_{i} \chi_{E_{i}}$, be a step function. We define $\mu$-integral $\int_{X} \varphi d \mu$ of $\varphi$ over $X$ by $\sum_{i=1}^{n} y_{i} \mu\left(E_{i}\right)$.

Definition 1.8 ([3]). A $\mu$-measurable function $\varphi: X \rightarrow Y$ is called Bochner integrable if there exists a sequence of step functions $\left(\varphi_{n}\right)_{n \in \mathbb{N}}$ such that

$$
\lim _{n \rightarrow \infty} \int_{X}\left\|\varphi_{n}-\varphi\right\| d \mu=0 \text {. }
$$




\section{ON DIFFERENTIABILITY OF MAPPINGS WITH FINITE DILATION}

In this case, the integral $\int_{E} \varphi d \mu$ is defined uniquely for each $E \in \Sigma$ in the following way

$$
\int_{E} \varphi d \mu=\lim _{n \rightarrow \infty} \int_{E} \varphi_{n} d \mu
$$

TheOREM $1.9([3])$. A $\mu$-measurable function $\varphi: X \rightarrow Y$ is Bochner integrable if and only if $\int_{X}\|\varphi\| d \mu<\infty$.

Remark 1.10 (1]). For measurable sets in $\mathbb{R}^{k}$, the Bochner integral of real functions with respect to Lebesgue measure coincides with Lebesgue integral.

Theorem 1.11 ([3]). Let $\varphi:(a, b) \rightarrow Y$ be Bochner integrable on $(a, b)$ with respect to Lebesgue measure. Then, for almost all $s \in(a, b)$,

$$
\lim _{h \rightarrow 0} \frac{1}{h} \int_{s}^{s+h}\|\varphi(t)-\varphi(s)\| d t=0
$$

Consequently, for almost all $s \in(a, b)$,

$$
\lim _{h \rightarrow 0} \frac{1}{h} \int_{s}^{s+h} \varphi(t) d t=\varphi(s) .
$$

Definition 1.12 ([8]). We say that a mapping $\varphi:(a, b) \rightarrow \mathbb{R}$ is absolutely continuous on $(a, b), \varphi \in A C(a, b)$ if

$\forall \varepsilon>0 \exists \delta>0 \forall\left(a_{1}, b_{1}\right), \ldots,\left(a_{n}, b_{n}\right): \sum_{i=1}^{n}\left(b_{i}-a_{i}\right)<\delta \Rightarrow \sum_{i=1}^{n}\left|\varphi\left(b_{i}\right)-\varphi\left(a_{i}\right)\right|<\varepsilon$.

Definition 1.13 ([6]). We say that an additive mapping $\varphi: \Sigma \rightarrow Y$ is absolutely continuous on $X, \varphi \in A C(X)$ if

$$
\forall \varepsilon>0 \exists \delta>0 \forall A \in \Sigma: \mu(A)<\delta \Rightarrow\|\varphi(A)\|<\varepsilon .
$$

Theorem 1.14 ([6]). If $\varphi: X \rightarrow Y$ is integrable, then $\Phi: \Sigma \rightarrow Y, \Phi(E)=$ $\int_{E} \varphi d \mu$ for $E \in \Sigma$, is absolutely continuous on $X$.

Corollary 1.15. If $X=(a, b)$ and $\varphi: \Sigma \rightarrow Y$ is absolutely continuous on $(a, b)$ and $x_{0} \in(a, b)$ is fixed, then the mapping $\Phi:(a, b) \rightarrow Y$,

$$
\Phi(x)= \begin{cases}\varphi\left(\left[x_{0}, x\right]\right), & x \geqslant x_{0} \\ \varphi\left(\left[x, x_{0}\right]\right), & x<x_{0}\end{cases}
$$

is absolutely continuous on $(a, b)$.

Lemma 1.16. Let $g:(a, b) \rightarrow Y$ be a mapping. Assume that for each $y^{*} \in Y^{*}$, the mapping $y^{*} \circ g:(a, b) \rightarrow \mathbb{R}$ is constant. Then, $g$ is constant. 
Proof. Suppose that the mapping $g$ is not constant. There exist $t_{1}, t_{2}$, $a<t_{1}<t_{2}<b$, such that $g\left(t_{1}\right) \neq g\left(t_{2}\right)$. By Hahn-Banach theorem, there exists a functional $y^{*} \in Y^{*}$ such that $y^{*}\left(g\left(t_{1}\right)-g\left(t_{2}\right)\right) \neq 0$. Therefore, $\left(y^{*} \circ g\right)\left(t_{1}\right) \neq$ $\left(y^{*} \circ g\right)\left(t_{2}\right)$, a contradiction.

\section{Main result}

In this section, we will show that if the contingent is not trivial at each point of $\operatorname{Gr}(f)$, then $f$ is almost everywhere differentiable provided that $f$ satisfies some simple conditions (e.g., Lipschitz, finite dilation).

Let us consider the following question. Must the mapping $f:(a, b) \rightarrow Y$, $Y$ normed space, $\operatorname{dim} Y=\infty$, with finite dilation be almost everywhere differentiable?

Professor B. K i r ch h e i m suggested to investigate the following example.

EXAMPLE 2.1. There is a nowhere differentiable mapping with finite dilation and with trivial contingent at every point. Let us consider $K:(0,1) \rightarrow L^{1}[0,1]$,

$$
K(t):=\chi_{[0, t]} \cdot
$$

Finite dilation is evident from the definition of $K$, since

$$
\left\|K\left(t_{1}\right)-K\left(t_{2}\right)\right\|=\left\|\chi_{\left(t_{1}, t_{2}\right]}\right\|=\int_{t_{1}}^{t_{2}} d t=t_{2}-t_{1} \quad \text { for } 0<t_{1}<t_{2}<1 .
$$

Next, we will show that $K$ is nowhere differentiable indeed. Suppose to the contrary that there exists a point $t \in(0,1)$ and there exists a mapping $y \in L^{1}[0,1]$ such that $K^{\prime}(t)=y$. In other words,

$$
\int_{0}^{1}\left|\frac{K(t+h)(\tau)-K(t)(\tau)}{h}-y(\tau)\right| d \tau \rightarrow 0 \quad \text { when } \quad h \rightarrow 0 .
$$

First, assume that $h>0$. We have

$$
\begin{aligned}
\int_{0}^{1}\left|\frac{K(t+h)(\tau)-K(t)(\tau)}{h}-y(\tau)\right| d \tau & =\int_{0}^{1}\left|\frac{1}{h} \chi_{(t, t+h]}(\tau)-y(\tau)\right| d \tau \\
& =\int_{t}^{t+h}\left|\frac{1}{h}-y(\tau)\right| d \tau+\int_{[0,1] \backslash(t, t+h]}|y(\tau)| d \tau \\
& \geq \int_{[0,1]}|y(\tau)| d \tau .
\end{aligned}
$$




\section{ON DIFFERENTIABILITY OF MAPPINGS WITH FINITE DILATION}

This yields, by (2.1), that

$$
\int_{[0,1] \backslash(t, t+h]}|y(\tau)| d \tau \rightarrow 0 \quad \text { when } \quad h \rightarrow 0 .
$$

It follows that $y=0$ almost everywhere on $[0,1]$. Therefore,

$$
\int_{0}^{1}\left|\frac{1}{h} \chi_{(t, t+h]}(\tau)-y(\tau)\right| d \tau=\int_{0}^{1}\left|\frac{1}{h} \chi_{(t, t+h]}(\tau)\right| d \tau=\frac{1}{h} \int_{t}^{t+h} d \tau=1
$$

which contradicts (2.1). The case $h<0$ is similar. Hence, $K:(0,1) \rightarrow L^{1}[0,1]$ is nowhere differentiable.

Now, we will check that the contingent of the graph of $K$ is trivial at each point $(t, K(t))$, which means that it is zero vector $\left(0,0_{L^{1}[0,1]}\right)$ of $\mathbb{R} \times L^{1}[0,1]$.

Let

Clearly, $\left(0,0_{L^{1}[0,1]}\right) \in \operatorname{Tan}(\operatorname{Gr}(K),(t, K(t)))$ for each $t \in(0,1)$. Fix $t \in(0,1)$.

$$
(\alpha, w) \in \operatorname{Tan}(\operatorname{Gr}(K),(t, K(t))), \quad(\alpha, w) \in \mathbb{R} \times L^{1}[0,1] .
$$

Then, by Definition 1.1, there exist sequences

$$
\left(t_{n}\right)_{n \in \mathbb{N}}, \quad t_{n} \in(0,1), \quad t_{n} \rightarrow t, \quad \text { and } \quad\left(\lambda_{n}\right)_{n \in \mathbb{N}}, \quad \lambda_{n}>0,
$$

such that

$$
\lambda_{n}\left(t_{n}-t, K\left(t_{n}\right)-K(t)\right) \rightarrow(\alpha, w) \quad \text { in } \quad \mathbb{R} \times L^{1}[0,1] .
$$

First, assume that $t_{n}>t$. Then, $K\left(t_{n}\right)-K(t)=\chi_{\left(t, t_{n}\right]}$. Therefore,

$$
\lambda_{n}\left(K\left(t_{n}\right)-K(t)\right)=\lambda_{n} \chi_{\left(t, t_{n}\right]} \rightarrow w \quad \text { in } \quad L^{1}[0,1],
$$

i.e.,

$$
\lim _{n \rightarrow \infty} \int_{0}^{1}\left|\lambda_{n} \chi_{\left(t, t_{n}\right]}(\xi)-w(\xi)\right| d \xi=0
$$

On the other hand, for each $n \in \mathbb{N}$, we get

$$
\begin{aligned}
\int_{0}^{1}\left|\lambda_{n} \chi_{\left(t, t_{n}\right]}(\xi)-w(\xi)\right| d \xi & =\int_{\left(t, t_{n}\right]}\left|\lambda_{n} \chi_{\left(t, t_{n}\right]}(\xi)-w(\xi)\right| d \xi+\int_{[0,1] \backslash\left(t, t_{n}\right]}|w(\xi)| d \xi \\
& \geq \int_{[0,1] \backslash\left(t, t_{n}\right]}|w(\xi)| d \xi,
\end{aligned}
$$

whence by (2.2),

$$
\int_{[0,1] \backslash\left(t, t_{n}\right]}|w(\xi)| d \xi \rightarrow 0 \quad \text { as } \quad n \rightarrow \infty
$$


Since

$$
\lim _{n \rightarrow \infty} \int_{[0,1] \backslash\left(t, t_{n}\right]}|w(\xi)| d \xi=\int_{0}^{1}|w(\xi)| d \xi
$$

we obtain $w=0_{L^{1}[0,1]}$ almost everywhere, and therefore

$$
\int_{0}^{1}\left|\lambda_{n} \chi_{\left(t, t_{n}\right]}(\xi)-w(\xi)\right| d \xi=\int_{0}^{1}\left|\lambda_{n} \chi_{\left(t, t_{n}\right]}(\xi)\right| d \xi=\int_{\left(t, t_{n}\right]} \lambda_{n} d \xi=\lambda_{n}\left(t_{n}-t\right) \rightarrow \alpha .
$$

It follows by (2.2) that $\alpha=0$.

The case $t_{n}<t$ is treated analogously and yields the same result. We conclude that $(\alpha, w)=\left(0,0_{L^{1}[0,1]}\right)$. Thus, $\operatorname{Tan}(\operatorname{Gr}(K),(t, K(t)))$ is trivial for each $t \in(0,1)$.

Theorem 2.2. Let $Y$ be a separable Banach space and $\operatorname{dim} Y=\infty$. Assume that $f:(a, b) \rightarrow Y$ satisfies conditions:

(1) there exists a Lebesgue integrable mapping $g:(a, b) \rightarrow[0, \infty)$ such that for each $t \in(a, b)$,

$$
\limsup _{h \rightarrow 0}\left\|\frac{f(t+h)-f(t)}{h}\right\| \leq g(t)
$$

(2) for each $t \in(a, b)$, the contingent $\operatorname{Tan}(\operatorname{Gr}(f),(t, f(t)))$ is a one-dimensional vector subspace of $\mathbb{R} \times Y$.

Under these assumptions, $f$ is almost everywhere differentiable.

P r o of. Let $t \in(a, b)$ and assume that $(\alpha(t), w(t)) \in \operatorname{Tan}(\operatorname{Gr}(f),(t, f(t)))$ is a non-zero vector. Such a vector exists by assumption that the contingent is not trivial. By Definition 1.1 there exists a sequence $\left(t_{n}\right)_{n \in \mathbb{N}}, t_{n} \in(a, b), t_{n} \rightarrow t$, and there exists a sequence $\left(\lambda_{n}\right)_{n \in \mathbb{N}}, \lambda_{n}>0$, such that $\lambda_{n}\left(t_{n}-t\right) \rightarrow \alpha(t)$ and $\lambda_{n}\left(f\left(t_{n}\right)-f(t)\right) \rightarrow w(t)$. It is impossible to have $\alpha(t)=0, w(t) \neq 0_{Y}$. This follows at once from condition (1) because

$$
\lambda_{n}\left(f\left(t_{n}\right)-f(t)\right)=\lambda_{n}\left(t_{n}-t\right) \frac{f\left(t_{n}\right)-f(t)}{t_{n}-t} \rightarrow w(t) .
$$

Since $(\alpha(t), w(t)) \neq\left(0,0_{Y}\right)$, we have by (2.3) that there exists

$$
\lim _{n \rightarrow \infty} \frac{f\left(t_{n}\right)-f(t)}{t_{n}-t}=\frac{w(t)}{\alpha(t)} .
$$

Set $v(t)$ to denote this vector $\frac{w(t)}{\alpha(t)}$. Note that, since dim $\operatorname{Tan}(\operatorname{Gr}(f),(t, f(t)))=1$, it is clear that $v(t)$ is defined uniquely for each $t \in(a, b)$ and we have

$$
\operatorname{Tan}(\operatorname{Gr}(f),(t, f(t)))=\{\xi(1, v(t)): \xi \in \mathbb{R}\} .
$$




\section{ON DIFFERENTIABILITY OF MAPPINGS WITH FINITE DILATION}

Next, we will show that the mapping $v:(a, b) \rightarrow Y$ is Bochner integrable. Let $y^{*} \in Y^{*}$. It follows by (1) that

$$
\forall t \in(a, b): \limsup _{h \rightarrow 0}\left|\frac{\left(y^{*} \circ f\right)(t+h)-\left(y^{*} \circ f\right)(t)}{h}\right| \leq\left\|y^{*}\right\| g(t)<\infty .
$$

Since $y^{*} \circ f$ is real-valued, it is differentiable almost everywhere on $(a, b)$. Let $y^{*} \circ f$ be differentiable at $t \in(a, b)$, and $\left(t_{n}\right)_{n \in \mathbb{N}}$ be a sequence, $t_{n} \in(a, b), t_{n} \rightarrow t$, such that (cf. (2.4) )

$$
v(t)=\lim _{n \rightarrow \infty} \frac{f\left(t_{n}\right)-f(t)}{t_{n}-t} .
$$

Then, clearly,

$$
y^{*}(v(t))=\lim _{n \rightarrow \infty} y^{*}\left(\frac{f\left(t_{n}\right)-f(t)}{t_{n}-t}\right)=\left(y^{*} \circ f\right)^{\prime}(t) .
$$

Therefore,

$$
\forall y^{*} \in Y^{*}:\left(y^{*} \circ f\right)^{\prime}(t)=y^{*}(v(t)) \text { almost everywhere on }(a, b) .
$$

Thus, $y^{*} \circ f$ satisfies Lusin condition (N) (by (2.6) and Theorem 4.6 from [8]) which yields together with (1) and Theorem 7.7 from [8] that

$$
\forall y^{*} \in Y^{*}: y^{*} \circ f \in A C(a, b) .
$$

Due to (2.7), it follows that $y^{*} \circ v$ is measurable, hence $v$ is weakly measurable. Since $Y$ is separable and $v$ is weakly measurable, we get by Theorem 1.6 that the mapping $v$ is measurable, i.e., there exists a sequence $\left(v_{n}\right)_{n \in \mathbb{N}}$ of step functions $v_{n}:(a, b) \rightarrow Y$ taking a finite number of values such that

$$
\lim _{n \rightarrow \infty}\left\|v_{n}-v\right\|=0 \quad \text { almost everywhere on }(a, b) \text {. }
$$

It follows that $\left\|v_{n}\right\| \rightarrow\|v\|$ almost everywhere on $(a, b)$. However, for each $n \in \mathbb{N}$, the mapping $t \mapsto\left\|v_{n}(t)\right\|$ is measurable (it is a finitely-valued real function, whence $t \mapsto\|v(t)\|$ is measurable). By (1), we get $\|v(t)\| \leq g(t)$ for each $t \in(a, b)$, whence

$$
\int_{a}^{b}\|v(\tau)\| d \tau<\infty
$$

Therefore, $v:(a, b) \rightarrow Y$ is Bochner integrable (Theorem 1.9).

Fix $t_{0} \in(a, b)$ and consider the mapping $F:(a, b) \rightarrow Y$ defined by

$$
F(t)=-\int_{t_{0}}^{t} v(\tau) d \tau
$$


From Corollary 1.15, we obtain that $F$ is $A C(a, b)$, and from Theorem 1.11, we get, in view of (2.8), that $F^{\prime}(t)=-v(t)$ for almost all $t \in(a, b)$. Put $t \in(a, b)$ such that $F^{\prime}(t)$ exists and $F^{\prime}(t)=-v(t)$. Fix $y^{*} \in Y^{*}$. By (2.5), we have

$$
\operatorname{Tan}\left(\operatorname{Gr}\left(y^{*} \circ f\right),\left(t,\left(y^{*} \circ f\right)(t)\right)\right)=\left\{\xi\left(1, y^{*}(v(t))\right): \xi \in \mathbb{R}\right\} .
$$

Applying Lemma 1.2 for $y^{*} \circ F$ and $y^{*} \circ f$, we get

$$
\begin{aligned}
& \operatorname{Tan}\left(\operatorname{Gr}\left(y^{*} \circ F+y^{*} \circ f\right),\left(t,\left(y^{*} \circ F+y^{*} \circ f\right)(t)\right)\right) \\
& =\left\{\left(\alpha,\left(y^{*} \circ F\right)^{\prime}(t) \alpha+\beta\right):(\alpha, \beta) \in \operatorname{Tan}\left(\operatorname{Gr}\left(y^{*} \circ f\right),\left(t,\left(y^{*} \circ f\right)(t)\right)\right)\right\} \\
& =\left\{\left(\xi,-y^{*}(v(t)) \xi+y^{*}(v(t)) \xi\right): \xi \in \mathbb{R}\right\}=\{(\xi, 0): \xi \in \mathbb{R}\} .
\end{aligned}
$$

In other words, for each $y^{*} \in Y^{*}$,

$$
\operatorname{Tan}\left(\operatorname{Gr}\left(y^{*} \circ F+y^{*} \circ f\right),\left(t,\left(y^{*} \circ F+y^{*} \circ f\right)(t)\right)\right)=\{(\xi, 0): \xi \in \mathbb{R}\}
$$

for almost all $t \in(a, b)$.

Therefore, for each $y^{*} \in Y^{*}$, we get that $y^{*} \circ(F+f)$ is $A C(a, b)$ and

$$
\left(y^{*} \circ(F+f)\right)^{\prime}(t)=0 \text { for almost each } t \in(a, b) \text {. }
$$

It follows that the mapping $y^{*} \circ(F+f)$ is constant for each $y^{*} \in Y^{*}$. By Lemma 1.16, $F+f$ is constant. Since $F$ is almost everywhere differentiable, we conclude that so is $f$.

Remark 2.3. In particular, if $f$ is Lipschitz (in Theorem 2.2) and (2) is fulfilled, then condition (1) is obviously satisfied, whence $f$ is almost everywhere differentiable and $\|v\|$ is bounded. Also observe that the mapping $g$ (in Theorem 2.2) may be considered only locally integrable.

\section{REFERENCES}

[1] ALEXIEWICZ, A.: Analiza Funkcjonalna. Państwowe Wydawnictwo Naukowe, Warszawa, 1969. (In Polish)

[2] BOULIGAND, G.: Introduction à la Géométrie Infinitésimale Directe. Librairie Vuibert, Paris, 1932.

[3] Diestel, J.-UHL, J. J.: Vector Measures, in: Math. Surveys Monogr., Vol. 15, Amer. Math. Soc., Providence, R.I., 1977.

[4] FEDERER, H.: Geometric Measure Theory, in: Die Grundlehren der Mathematischen Wissenschaften in Einzeldarstellungen, Vol. 153, Springer-Verlag, Berlin, 1969.

[5] FLETT, T. M.: Differential Analysis. Cambridge University Press, London, 1980.

[6] PETTIS, B. J.: On integration in vector spaces, Trans. Amer. Math. Soc. 44 (1938), 277-304. 


\section{ON DIFFERENTIABILITY OF MAPPINGS WITH FINITE DILATION}

[7] PONOMAREV, S.-TUROWSKA, M.: Lipschitz mappings, contingents, and differentiability, Siberian Math. J. 48 (2007), 669-677.

[8] SAKS, S.: Theory of the Integral. G. E. Stechert \& Co., New York, 1937.

[9] SCHWARTZ, L.: Analyse Mathématique, Vol. 1, Hermann, Paris, 1967.

Received October 29, 2014

Institute of Mathematics

Pomeranian University in Stupsk

ul. Arciszewskiego 22d

PL-76-200 Stupsk

POLAND

E-mail: malgorzata.turowska@apsl.edu.pl 